\title{
Low Vitamin D Levels and Frailty Status in Older Adults: A Systematic Review and Meta-Analysis
}

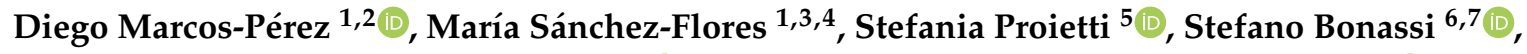

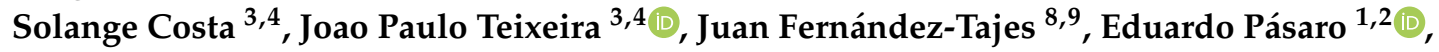 \\ Vanessa Valdiglesias $2,4,10, *,+$ and Blanca Laffon $1,2,+$ D \\ 1 Grupo DICOMOSA, Centro de Investigaciones Científicas Avanzadas (CICA), Departamento de Psicología, \\ Facultad de Ciencias de la Educación, Campus Elviña s/n, Universidade da Coruña, 15071 A Coruña, Spain; \\ diego.marcos@udc.es (D.M.-P.); maria.sanchez@udc.es (M.S.-F.); pspasaro@udc.es (E.P.); \\ blaffon@udc.es (B.L.) \\ 2 Instituto de Investigación Biomédica de A Coruña (INIBIC), AE CICA-INIBIC, Oza, 15071 A Coruña, Spain \\ 3 Environmental Health Department, National Health Institute, Rua Alexandre Herculano 321, 4000-055 Porto, \\ Portugal; solange.costa2@gmail.com (S.C.); jpft12@gmail.com (J.P.T.) \\ 4 EPIUnit-Instituto de Saúde Pública, Universidade do Porto, Rua das Taipas, no 135, 4050-600 Porto, Portugal \\ 5 Scientific Direction, IRCCS San Raffaele Pisana, Via di Val Cannuta, 247, 00166 Rome, Italy; \\ stefania.proietti@sanraffaele.it \\ 6 Unit of Clinical and Molecular Epidemiology, IRCCS San Raffaele Pisana, Via di Val Cannuta, 247, \\ 00166 Rome, Italy; stefano.bonassi@sanraffaele.it \\ 7 Department of Human Sciences and Quality of Life Promotion, San Raffaele University, Via di Val Cannuta, \\ 247, 00166 Rome, Italy \\ 8 Wellcome Centre for Human Genetics, McCarthy's group, University of Oxford, Roosevelt Drive, \\ Headington, Oxford OX3 7BN, UK; jfertaj@well.ox.ac.uk \\ 9 Genetic and Molecular Epidemiology Unit, Department of Clinical Sciences, CRC, SUS Malmö, \\ Jan Waldenströms gata 35, House 91:12, SE-214 28 Malmö, Sweden \\ 10 Grupo DICOMOSA, Centro de Investigaciones Científicas Avanzadas (CICA), Departamento de Biología, \\ Facultad de Ciencias, Campus A Zapateira s/n, Universidade da Coruña, 15071 A Coruña, Spain \\ * Correspondence: vvaldiglesias@udc.es; Tel.: +34-881-012680 \\ + These authors contributed equally to the senior authorship of this manuscript.
}

Received: 22 May 2020; Accepted: 24 July 2020; Published: 30 July 2020

Abstract: Serum vitamin D deficiency is widespread among older adults and is a potential modifiable risk factor for frailty. Moreover, frailty has been suggested as an intermediate step in the association between low levels of vitamin D and mortality. Hence, we conducted a systematic review of the literature and meta-analysis to test the possible association of low concentrations of serum 25-hydroxyvitamin $\mathrm{D}(25(\mathrm{OH}) \mathrm{D})$, a marker of vitamin D status, with frailty in later life. We reviewed cross-sectional or longitudinal studies evaluating populations of older adults and identifying frailty by a currently validated scale. Meta-analyses were restricted to cross-sectional data from studies using Fried's phenotype to identify frailty. Twenty-six studies were considered in the qualitative synthesis, and thirteen studies were included in the meta-analyses. Quantitative analyses showed significant differences in the comparisons of frail (standardized mean difference (SMD)-1.31, 95\% confidence interval (CI) $(-2.47,-0.15), p=0.0271)$ and pre-frail (SMD-0.79, 95\% CI $(-1.58,-0.003)$, $p=0.0491$ ) subjects vs. non-frail subjects. Sensitivity analyses reduced heterogeneity, resulting in a smaller but still highly significant between-groups difference. Results obtained indicate that lower 25(OH)D levels are significantly associated with increasing frailty severity. Future challenges include interventional studies testing the possible benefits of vitamin D supplementation in older adults to prevent/palliate frailty and its associated outcomes.

Keywords: frailty; meta-analysis; older adults; systematic review; vitamin D 


\section{Introduction}

Vitamin D is a fat soluble secosteroid hormone which is mainly produced endogenously in the skin (80-90\%) after sunshine exposure. The other sources of vitamin D (10-20\%) are the intake of food which naturally contains this vitamin and dietary supplements [1]. Vitamin D has a plethora of functions; the major physiologic function is regulating calcium and phosphate homeostasis as well as mineral bone metabolism. However, vitamin D also plays an extensive role as a cell differentiating and antiproliferative factor with actions in a variety of tissues, including the renal, cardiovascular, and immune systems [2,3].

Although biologically inert, 25-hydroxyvitamin D $(25(\mathrm{OH}) \mathrm{D})$ is the major circulating metabolite of vitamin $\mathrm{D}$ and is globally accepted as a marker of vitamin $\mathrm{D}$ status. There is no consensus about the cut-offs of this metabolite to describe an individual's vitamin D status. Thus, the Institute of Medicine and the Endocrine Society have different definitions for vitamin D deficiency and optimal values $[4,5]$. The prevalence of serum 25(OH)D levels lower than $20 \mathrm{ng} / \mathrm{mL}$ in the US population was reported as $32 \%$ [6], and concentrations lower than $30 \mathrm{ng} / \mathrm{mL}$ were observed in $57.5 \%$ of men and in $60.7 \%$ of women in a Canadian cohort [7]. Variations in prevalence may be related to characteristics of the analyzed populations such as sex, age, skin pigmentation, or vitamin D supplementation, or even to the sampling season $[6,7]$.

The prevalence of vitamin D deficiency is higher in older adults than in younger adults, and especially higher in institutionalized (30-90\%) than in community-dwelling older people $(2-60 \%)[8,9]$. There are multiple causes of this higher prevalence in later life, including lower sunshine exposure owing to a reduction in outdoor activity, lower dermal synthesis of pre-vitamin $\mathrm{D}$, inadequate vitamin $\mathrm{D}$ intake, and decreased kidney function (calcitriol, the biologically active form of vitamin $\mathrm{D}$, is produced in the kidney proximal tubule) [10].

The older population is particularly at risk for clinical complications associated with vitamin $\mathrm{D}$ deficiency. Several cross-sectional studies linked low vitamin D status with an increased risk of type 1 and type 2 diabetes mellitus, cardiovascular disease, certain cancers, cognitive decline, depression, autoimmunity, allergy, and even frailty [1]. Frailty is a geriatric syndrome with multiple causes and contributors, characterized by diminished strength, endurance, and reduced physiologic function, which increases an individual's vulnerability for developing negative health outcomes, increased dependency and/or death [11]. A plethora of frailty measurements are currently in existence; among them, the two frailty identification tools most commonly accepted and used in clinical settings and in research studies are the phenotypic model proposed by Fried et al. [12] and the deficit accumulation model developed by Mitnitski, Mogilner, and Rockwood [13]. Prevalence of frailty is highly variable and dependent on a number of factors (e.g., frailty identification criteria, gender, age or socio-economic conditions); the heterogeneity of factors associated with frailty is reflected by the wide variation of prevalence estimates, ranging from $4 \%$ to $59.1 \%$ [14].

A potential modifiable risk factor for frailty seems to be serum vitamin D deficiency which, as already mentioned, is widespread among older adults. Moreover, frailty has been suggested as an intermediate step in the association between low levels of vitamin D and mortality [15]. A clear inverse association between vitamin $\mathrm{D}$ level and frailty in older adult populations has been reported in several studies [16-18], although others did not find such association [19,20].

The diversity of: (i) frailty model components, (ii) definitions of vitamin D deficiency, and (iii) laboratory methods used to measure $25(\mathrm{OH}) \mathrm{D}$ may in part explain the divergence of findings among studies examining the association of vitamin D status with frailty and mortality. For these reasons, and in order to confirm the hypothesis that vitamin D deficiency may be related to frailty syndrome, we conducted a systematic review of the literature and meta-analysis to test the possible association of low levels of $25(\mathrm{OH}) \mathrm{D}$ with frailty status in later life. Two previous meta-analyses have been published exploring this association [21,22]. These studies included a smaller number of studies (four cross-sectional plus six longitudinal studies, and seven longitudinal studies, respectively), did not consider the condition of pre-frailty, and did not take into consideration a major source of variability 
such as the frailty identification criteria used in the original studies. For these reasons, together with the inclusion of additional recent studies analyzing vitamin D in frail subjects, a newly updated, more comprehensive and more homogeneous meta-analysis is required, which may provide quantitative indication about the level of $25(\mathrm{OH}) \mathrm{D}$ in frailty patients and in early stages of this syndrome.

\section{Materials and Methods}

The Preferred Reporting Items from Systematics Reviews and Meta-Analyses (PRISMA) statement [23] was followed to perform and report the present systematic review and meta-analyses.

\subsection{Eligibility Criteria for the Systematic Review}

Cross-sectional or longitudinal design observational studies conducted in humans, including populations of older adults ( $\geq 60$ years old), and written in English or Spanish were considered eligible for this systematic review. In these studies, participant's frailty had to be identified according to any of the currently validated scales for frailty identification. Review articles, editorials, commentaries, letters without data analysis, conference abstracts, studies conducted in animals, experimental studies, studies assessing populations with severe pathologies (cancer, diabetes, heart failure, etc.), considering frailty as a confounder, or employing a non-validated frailty identification tool were excluded.

\subsection{Search Strategy}

Studies included in this systematic review were identified through an extensive bibliographic search using the PubMed database (National Library of Medicine, National institutes of Health, Berthesda, MD, USA; http://www.ncbi.nih.gov/PubMed), updated to October 2018. The search was conducted by two independent researchers (D.M.-P. and V.V.) following a search strategy which comprised two terms intersected using the Boolean term "AND". The search term included as the first descriptor was related to frailty ("frail*"), and the second one included descriptors related to vitamin D ("vitamin D" or "25(OH)D"). The search filter "Humans" was used to retrieve only studies conducted in human subjects. Initial screening was focused on title or abstract.

\subsection{Data Collection Process}

The following information was collected independently by two authors (D.M.-P. and V.V.) for each study complying with inclusion criteria: country of origin for the first author, type of study, size of the study population and the frailty groups, participant's gender and age, criteria used to identify frailty, and outcomes (circulating concentrations of $25(\mathrm{OH}) \mathrm{D}$ ) in each frailty group.

\subsection{Studies Included in the Meta-Analysis}

Studies included in the meta-analysis were those providing mean \pm standard deviation (SD) data of $25(\mathrm{OH}) \mathrm{D}$ for frailty groups. Several studies provided quantitative parameters different than the mean, e.g., median and confidence intervals (CI) or inter-quartile range, or they classified the population according to $25(\mathrm{OH}) \mathrm{D}$ concentration ranges instead of by frailty status. In all these cases, emails were sent to corresponding authors asking for missing values, at least twice in a four-month period. Studies for which authors provided the requested data were also included in the meta-analyses. Meta-analyses were restricted to only those studies employing Fried's criteria (original or modified version) to identify frailty. Only cross-sectional data were considered for the meta-analyses, since just six longitudinal studies which employed different frailty identification tools were available; hence, in longitudinal studies, just baseline values of $25(\mathrm{OH}) \mathrm{D}$ were included (follow-up values were dismissed).

\subsection{Quality Assessment}

Quality assessment of studies included in the meta-analyses was carried out by two independent investigators (D.M-.P. and V.V.), whilst a third reviewer was available for mediation (B.L.). The standard 
of study design was evaluated for each study by calculating a quality score (Q.S.) (Supplementary Material, Table S1). Each QS item scored from 1 to a maximum of 3 points based on reported data and matching status. The minimum and maximum of total QS possible was five and seventeen, respectively.

\subsection{Statistical Analysis}

All analyses were performed using the Comprehensive R Archive Network (https://cran.r-project. org/). Data were expressed as mean $25(\mathrm{OH}) \mathrm{D} \pm \mathrm{SD}$. Heterogeneity across studies was evaluated using the $\mathrm{I}^{2}$ and tested with the Cochran $\mathrm{Q}$ chi-square statistics. Since all meta-analyses conducted showed statistically significant heterogeneity among studies $\left(\mathrm{I}^{2} \geq 50 \%\right.$ and $p$ value $\left.<0.05\right)$, the pooled standardized mean differences (SMD) with $95 \%$ confidence interval $(95 \% \mathrm{CI})$ were estimated using a random-effects model with method according to Dersimonian and Laird [24]. Publication bias was assessed by visually inspecting funnel plots and using the Egger's bias test. Whenever a significant result $(p<0.05)$ was found, the trim-and-fill method was used to adjust for any potential unpublished studies. The presence of confounders or effect modifiers was tested with a meta-regression analysis; the final model included year of publication and QS (as a continuous variable) as confounders. A sensitivity analysis was performed when a discrepant value of SMD in a single study was observed [25].

\section{Results}

\subsection{Literature Search Results}

Two hundred and sixty-nine articles were initially identified in the literature search. After removal of duplicates, 218 articles were screened for potential eligibility (a flow chart is shown in Figure 1). Titles and abstracts were reviewed and 84 out of 218 were retrieved for full-text assessment of eligibility. Then, whole texts were completely reviewed, obtaining 19 studies which fitted the selection criteria. Seven additional publications were incorporated from the references section of published articles. Eventually, 26 studies fulfilled the inclusion criteria and were included in this review. After excluding studies not reporting mean + SD data or not employing Fried's criteria for frailty identification, 13 studies were finally included in the meta-analysis.

\subsection{Characteristics of Included Studies}

Table 1 shows the characteristics of the 26 studies included in the systematic review (total sample size: 38,162 participants). None of the retrieved studies written in Spanish fulfilled the inclusion criteria. Eight out of 10 studies were performed with sample sizes above 300 participants, having a relatively high statistical power. Globally, percentages of individuals included in the three different frailty groups were $46.5 \%$ non-frail, $38 \%$ pre-frail and $15.5 \%$ frail participants. Prevalence of frailty and pre-frailty in the different studies ranged between $4 \%$ and $58 \%$, and between $21 \%$ and $63 \%$, respectively. Four studies reported both cross-sectional and longitudinal prospective evaluations (follow up periods from 2.9 to 12 years). Seven studies included females only, six studies included males only, and 13 included both genders. Europe and the USA were the main places where these studies were performed, with $46 \%$ and $35 \%$ of the total participants, respectively, followed by Asia and Australia (8\% each), and only one study performed in Mexico. Focusing on European countries, four studies were conducted in Spain, two in Germany and the UK, and only one in the Netherlands, Portugal, Italy and France. According to the methodology employed to measure $25(\mathrm{OH}) \mathrm{D}$, the radioreceptor assay, including radioimmunoassay, was used in half of the studies, while $15 \%$ of studies employed a chemiluminiscence assay. Frailty status was identified by using Fried's criteria (original version or modified) in $80 \%$ of studies. Among them, $54 \%$ used the original version of frailty phenotype, and the rest of them introduced different modifications.

The minimum and maximum QS obtained in the studies included in the meta-analyses were seven and twelve, respectively (Table S1). Only one study included between 100 and 300 subjects; all other studies had more than 300 participants. Half of the studies were matched for age (difference 
between groups was less than five years), or for gender (studies not matched for this characteristic had a gender ratio either higher than 0.60 or lower than 0.40 ). In only two studies, frailty groups were balanced, and none of the studies described whether frailty was measured by geriatricians or specialized personnel. Approximately half of the studies (six studies) comprised only non-frail and frail groups, while the other half (seven studies) included also a pre-frail group.

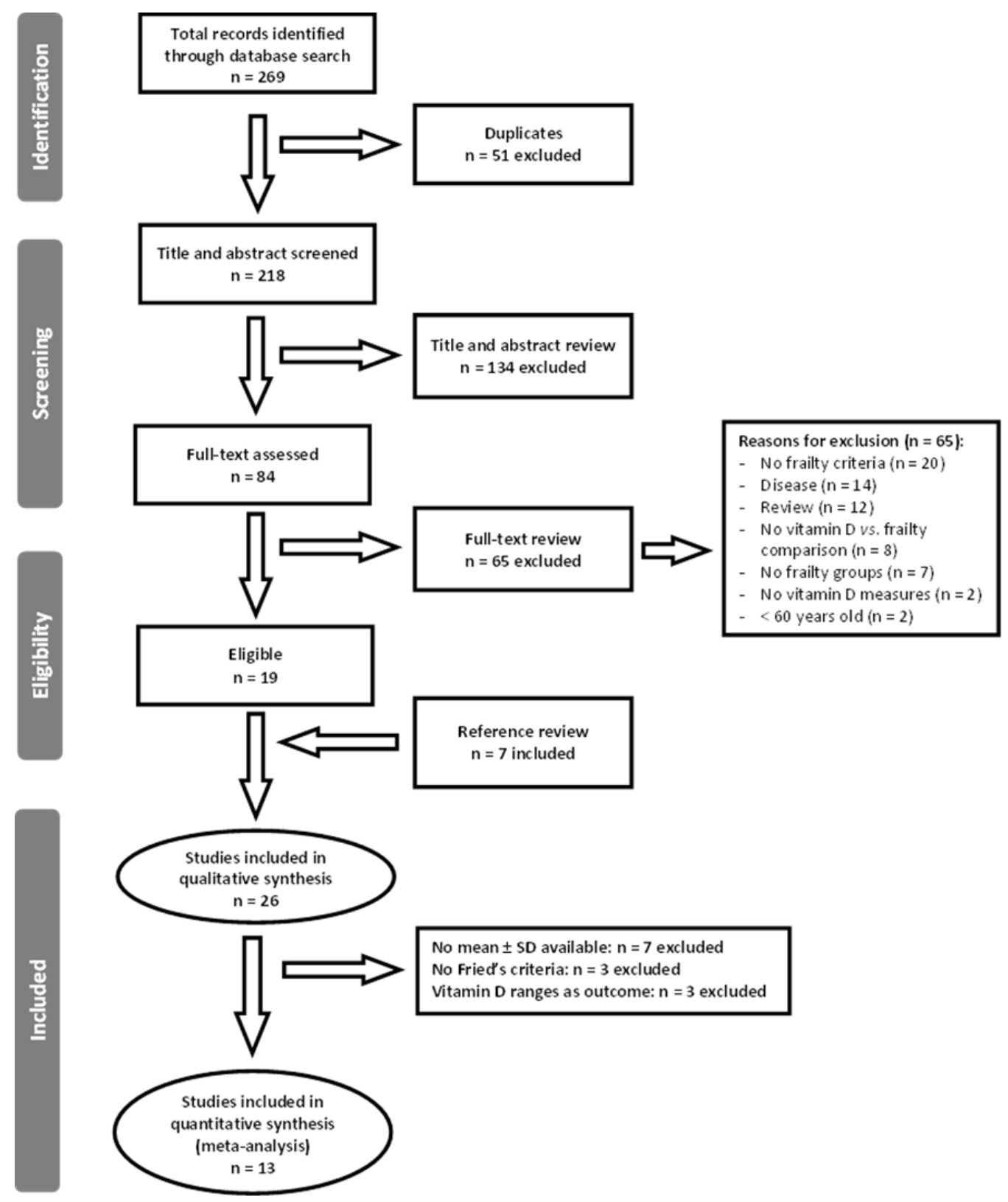

Figure 1. Preferred Reporting Items for Systematic Reviews and Meta-Analyses (PRISMA) flow chart of the study selection process. SD: standard deviation.

\subsection{Meta-Analysis Frailty Group vs. Non-Frailty Group}

This meta-analysis included 12 studies out of the 13 suitable (one of the selected studies did not include a frail group) (see forest plot in Figure 2a). Heterogeneity was very high, even after removing most extreme results $(96.05 \%, p<0.0001)$ (Table 2; Figure $2 b)$. No evidence of publication bias was found, and the concentration of $25(\mathrm{OH}) \mathrm{D}$ was significantly lower in the frailty group when compared to the non-frailty group. 
Table 1. Characteristics of the studies included in the systematic review. Studies in bold were included in the meta-analyses.

\begin{tabular}{|c|c|c|c|c|c|c|c|c|}
\hline Study & $\begin{array}{l}\text { First Author } \\
\text { Country }\end{array}$ & Study Design & $\begin{array}{c}\text { Population } \\
\text { (Mean Age } \pm \text { SD) } \\
\text { (years) }\end{array}$ & $\begin{array}{l}\text { Case Population } \\
\text { (Mean Age } \pm \text { SD) } \\
\text { (years) }\end{array}$ & $\begin{array}{c}\text { Control Population } \\
\text { (Mean Age } \pm \text { SD) } \\
\text { (years) }\end{array}$ & $\begin{array}{l}\text { Frailty } \\
\text { Criteria }\end{array}$ & $\begin{array}{l}\text { Outcome and Assay } \\
\text { Method }\end{array}$ & Results \\
\hline Alvarez-Ríos et al., 2015 [26] & Spain & Cross-sectional & $n=592$ female & $\begin{array}{c}n=61 \text { frail } \\
\text { (median 78, IQR } \\
75-83 \text { ) } \\
n=232 \text { pre-frail } \\
\text { (median 74, IQR } \\
71-78)\end{array}$ & $\begin{array}{c}n=299 \text { non-frail } \\
\text { (median 72, IQR } \\
\text { 69-76) }\end{array}$ & Fried's phenotype & $\begin{array}{l}25(\mathrm{OH}) \mathrm{D} \\
\text { electro-chemiluminescence }\end{array}$ & $\begin{array}{c}\downarrow 25(\mathrm{OH}) \mathrm{D} \text { in pre-frail and frail vs. } \\
\text { non-frail subjects }\end{array}$ \\
\hline Alvarez-Sánchez et al., 2018 [27] & Spain & Cross-sectional & $\begin{array}{c}n=631 \text { female } \\
\text { (median 74, IQR } \\
70-77 \text { ) }\end{array}$ & $\begin{array}{c}n=61 \text { frail } \\
n=245 \text { pre-frail }\end{array}$ & $n=325$ non-frail & Fried's phenotype & $\begin{array}{c}25(\mathrm{OH}) \mathrm{D} \\
\text { chemiluminescence } \\
\text { immunoassay }\end{array}$ & $\downarrow 25(\mathrm{OH}) \mathrm{D}$ with frailty \\
\hline \multirow[t]{2}{*}{ Chang et al., $2010[28]$} & \multirow{2}{*}{ Taiwan } & \multirow{2}{*}{ Cross-sectional } & \multirow{2}{*}{$n=215$} & $\begin{array}{c}n=21 \text { frail } \\
(72.1 \pm 4.4) \\
\text { male/female: } 6 / 15 \\
n=119 \text { pre-frail } \\
(71.4 \pm 3.8) \\
\text { male/female: } 49 / 70\end{array}$ & $\begin{array}{c}n=75 \text { non-frail } \\
\quad(70.3 \pm 3.7) \\
\text { male/female: } 32 / 43\end{array}$ & Fried's phenotype & \multirow{2}{*}{$\begin{array}{l}25(\mathrm{OH}) \mathrm{D} \\
\text { RIA }\end{array}$} & $\begin{array}{l}\downarrow 25(\mathrm{OH}) \mathrm{D} \text { with frailty using Fried's } \\
\text { phenotype }\end{array}$ \\
\hline & & & & $\begin{array}{c}n=26 \text { frail } \\
(71.9 \pm 3.7) \\
\text { male/female: } 7 / 19 \\
n=52 \text { pre-frail } \\
(71.5 \pm 3.6) \\
\text { male/female: } 14 / 38\end{array}$ & $\begin{array}{c}n=137 \text { non-frail } \\
\quad(70.8 \pm 4.0) \\
\text { male/female: } 66 / 71\end{array}$ & Edmonton Frail Scale & & $\begin{array}{l}\text { The association between } 25(\mathrm{OH}) \mathrm{D} \text { and } \\
\text { frailty is attenuated using the } \\
\text { Edmonton Frail Scale }\end{array}$ \\
\hline Ensrud et al., 2010 [16] & USA & $\begin{array}{l}\text { Cross-sectional } \\
\text { Longitudinal } \\
\text { (4.5-year } \\
\text { follow-up) }\end{array}$ & $\begin{array}{c}n=6307 \text { female }>69 \\
\quad \text { years old } \\
n=4551 \text { female }\end{array}$ & $\begin{array}{c}n=1065 \text { frail } \\
\text { (n.p.) } \\
n=3047 \text { pre-frail } \\
\text { (n.p.) }\end{array}$ & $\begin{array}{c}n=2195 \text { non-frail } \\
\text { (n.p.) } \\
n=4551 \text { non-frail } \\
\text { (non-frail + pre-frail) } \\
\text { (n.p.) }\end{array}$ & Fried's phenotype & $\begin{array}{l}\text { 25(OH)D } \\
\text { LC-MS/MS }\end{array}$ & $\begin{array}{l}\text { Lower }(<20 \mathrm{ng} / \mathrm{mL}) \text { and higher } \\
\text { ( } \geq 30 \mathrm{ng} / \mathrm{mL}) \text { levels of } 25(\mathrm{OH}) \mathrm{D} \text { were } \\
\text { moderately associated with higher } \\
\text { odds of frailty at baseline } \\
\text { Lower levels of } 25(\mathrm{OH}) \mathrm{D}(<20 \mathrm{ng} / \mathrm{mL}) \\
\text { were modestly associated with an } \\
\text { increased risk of incident } \\
\text { frailty or death at follow-up }\end{array}$ \\
\hline Ensrud et al., 2012 [29] & USA & Cross-sectional & $n=\begin{array}{c}1606 \text { male }>65 \\
\text { years old }\end{array}$ & $\begin{array}{c}n=130 \text { frail } \\
n=731 \text { pre-frail }\end{array}$ & $n=745$ non-frail & Fried's phenotype & $\begin{array}{l}\text { 25(OH)D } \\
\text { LC-MS/MS }\end{array}$ & $\uparrow \%$ frail male with $\downarrow 25(\mathrm{OH}) \mathrm{D}$ \\
\hline Fried et al., 2009 [30] & USA & Cross-sectional & $\begin{array}{c}n=704 \text { female } 70-79 \\
\text { years old }\end{array}$ & $\begin{array}{c}n=90 \text { frail } \\
n=330 \text { pre-frail }\end{array}$ & $n=284$ non-frail & Fried's phenotype & $\begin{array}{c}25(\mathrm{OH}) \mathrm{D} \\
\text { Radioreceptor assay }\end{array}$ & $\begin{array}{l}\downarrow 25(\mathrm{OH}) \mathrm{D} \text { with frailty (nearly } \\
\text { significant, } p=0.08 \text { ) }\end{array}$ \\
\hline $\begin{array}{l}\text { Gutierrez-Robledo et al., } \\
2015 \text { [31] }\end{array}$ & Mexico & Cross-sectional & $n=331$ & $\begin{array}{c}n=209 \text { frail } \\
(78.9 \pm 6.4) \\
\text { male/female: } 86 / 123\end{array}$ & $\begin{array}{c}n=122 \text { non-frail } \\
(79.9 \pm 4.7) \\
\text { male/female: } 66 / 56\end{array}$ & $\begin{array}{l}\text { Fried's phenotype } \\
\text { (modified) }\end{array}$ & $\begin{array}{l}\text { 25(OH)D } \\
\text { ELISA }\end{array}$ & $\begin{array}{l}\downarrow 25(\mathrm{OH}) \mathrm{D} \text { in frail vs. } \\
\text { non-frail subjects }\end{array}$ \\
\hline Hirani et al., 2013 [32] & Australia & Cross-sectional & $\begin{array}{l}n=1511 \text { male } \\
70-79 \text { years old }\end{array}$ & n.p & n.p & $\begin{array}{l}\text { Fried's phenotype } \\
\text { (modified) }\end{array}$ & $\begin{array}{l}25(\mathrm{OH}) \mathrm{D} \\
1,25 \mathrm{D} \\
\text { RIA }\end{array}$ & $\downarrow 25(\mathrm{OH}) \mathrm{D}$ and $1,25 \mathrm{D}$ with frailty \\
\hline Kojima \& Tanabe, 2016 [33] & UK & Cross-sectional & $\begin{array}{l}n=152 \mathrm{male} \\
(70.3 \pm 12.8)\end{array}$ & $\begin{array}{l}n=124 \text { frail } \\
(71.9 \pm 13.1)\end{array}$ & $\begin{array}{c}n=28 \text { non-frail } \\
(62.8 \pm 8.6)\end{array}$ & FI (34 items) & $\begin{array}{c}25(\mathrm{OH}) \mathrm{D} \\
\text { n.p }\end{array}$ & $\downarrow 25(\mathrm{OH})$ with frailty \\
\hline
\end{tabular}


Table 1. Cont.

\begin{tabular}{|c|c|c|c|c|c|c|c|c|}
\hline Study & $\begin{array}{l}\text { First Author } \\
\text { Country }\end{array}$ & Study Design & $\begin{array}{c}\text { Population } \\
\text { (Mean Age } \pm \text { SD) } \\
\text { (years) }\end{array}$ & $\begin{array}{c}\text { Case Population } \\
\text { (Mean Age } \pm \text { SD) } \\
\text { (years) }\end{array}$ & $\begin{array}{c}\text { Control Population } \\
\text { (Mean Age } \pm \text { SD) } \\
\text { (years) }\end{array}$ & $\begin{array}{l}\text { Frailty } \\
\text { Criteria }\end{array}$ & $\begin{array}{l}\text { Outcome and Assay } \\
\text { Method }\end{array}$ & Results \\
\hline Krams et al., 2016 [19] & France & Cross-sectional & $\begin{array}{c}n=321 \\
\text { (82.94 } 5.89) \\
\text { male/female: } \\
128 / 193\end{array}$ & $\begin{array}{c}n=146 \text { frail } \\
(84.10 \pm 5.77) \\
\text { male /female: } 54 / 92\end{array}$ & $\begin{array}{c}n=148 \text { non-frail } \\
(81.61 \pm 5.59) \\
\text { male/female: } 60 / 88\end{array}$ & $\begin{array}{l}\text { Fried's phenotype } \\
\text { (modified) }\end{array}$ & $\begin{array}{c}25(\mathrm{OH}) \mathrm{D} \\
\text { Chemiluminescence } \\
\text { immunoassay }\end{array}$ & $\begin{array}{l}25(\mathrm{OH}) \mathrm{D} \text { levels were not significantly } \\
\text { correlated with frailty }\end{array}$ \\
\hline Michelon et al., 2006 [34] & USA & Cross-sectional & $n=754$ female & $\begin{array}{c}n=86 \text { frail } \\
\text { (mean 75.8, 95\% CI } \\
75.1-76.5) \\
n=337 \text { pre-frail } \\
\text { (mean 74.4, 95\% CI } \\
74.1-74.7)\end{array}$ & $\begin{array}{c}n=331 \text { non-frail } \\
(\text { mean } 73.1,95 \% \text { CI } \\
73.7-74.4)\end{array}$ & Fried's phenotype & $\begin{array}{c}25(\mathrm{OH}) \mathrm{D} \\
\text { Radioreceptor assay }\end{array}$ & $\downarrow 25(\mathrm{OH}) \mathrm{D}$ with frailty \\
\hline Navarro-Martínez et al., 2016 [35] & Spain & Cross-sectional & $\begin{array}{l}n=104 \text { female } \\
(\text { mean }=84)\end{array}$ & $\begin{array}{c}n=60 \text { Frail } \\
n=22 \text { Pre-frail }\end{array}$ & $n=22$ non-frail & Fried's phenotype & $\begin{array}{l}25(\mathrm{OH}) \mathrm{D} \\
\mathrm{GC} / \mathrm{MS}\end{array}$ & $\begin{array}{l}\downarrow 25(\mathrm{OH}) \mathrm{D} \text { in pre-frail and frail vs. } \\
\text { non-frail subjects }\end{array}$ \\
\hline Pabst et al., 2015 [36] & Germany & Cross-sectional & $\begin{array}{c}n=940 \\
(75.6 \pm 6.5) \\
\text { male/female: } \\
478 / 762\end{array}$ & $\begin{array}{c}n=38 \text { Frail } \\
n=351 \text { Pre-frail }\end{array}$ & $n=551$ non-frail & Fried's phenotype & $\begin{array}{l}25(\mathrm{OH}) \mathrm{D} \\
\text { ECLIA System }\end{array}$ & $\downarrow 25(\mathrm{OH}) \mathrm{D}$ with frailty \\
\hline \multirow[b]{2}{*}{ Puts et al., 2005 [37] } & \multirow{2}{*}{$\begin{array}{l}\text { The } \\
\text { Netherlands }\end{array}$} & Cross-sectional & $n=1271$ & $\begin{array}{c}n=242 \text { frail } \\
(79.2 \pm 6.2) \\
\text { male/female: } 91 / 151\end{array}$ & $\begin{array}{c}n=1029 \text { non-frail } \\
(74.5 \pm 6.3) \\
\text { male/female: } 531 / 498\end{array}$ & \multirow[b]{2}{*}{ Nine frailty indicators } & \multirow{2}{*}{$\begin{array}{c}25(\mathrm{OH}) \mathrm{D} \\
\text { Competitive binding } \\
\text { protein assay }\end{array}$} & $\downarrow 25(\mathrm{OH}) \mathrm{D}$ with frailty \\
\hline & & $\begin{array}{l}\text { Longitudinal } \\
\text { (3-year } \\
\text { follow-up) }\end{array}$ & $n=885$ & $\begin{array}{c}n=125 \text { frail } \\
(78.2 \pm 6.2) \\
\text { male/female: } 56 / 69\end{array}$ & $\begin{array}{c}n=760 \text { non-frail } \\
(73.4 \pm 5.9) \\
\text { male/female: } 382 / 378\end{array}$ & & & $\downarrow 25(\mathrm{OH}) \mathrm{D}$ with frailty \\
\hline Sanchis et al., 2015 [38] & Spain & Cross-sectional & $\begin{array}{c}n=342 \\
\text { male/female: } \\
194 / 138\end{array}$ & $\begin{array}{c}n=116 \text { frail } \\
(81 \pm 7) \\
\text { male/female: } 47 / 69\end{array}$ & $\begin{array}{c}n=226 \text { non-frail } \\
(77 \pm 7) \\
\text { male/female: } 77 / 149\end{array}$ & Fried's phenotype & $\begin{array}{l}25(\mathrm{OH}) \mathrm{D} \\
\text { n.p. }\end{array}$ & $\begin{array}{c}\downarrow \text { vitamin } D \text { in frail vs. } \\
\text { non-frail subjects } \\
25(\mathrm{OH}) \mathrm{D}<9 \mathrm{ng} / \mathrm{mL} \text { was found as an } \\
\text { independent predictor of frailty }\end{array}$ \\
\hline Semba et al., 2006 [39] & USA & $\begin{array}{c}\text { Longitudinal } \\
\text { (3-year } \\
\text { follow-up) }\end{array}$ & $n=766$ female & $\begin{array}{l}n=250 \text { frail } \\
(80.4 \pm 7.9)\end{array}$ & $\begin{array}{c}n=516 \text { non-frail } \\
(76.1 \pm 7.4)\end{array}$ & Fried's phenotype & $\begin{array}{l}25(\mathrm{OH}) \mathrm{D} \\
\text { Radioreceptor assay }\end{array}$ & $\downarrow 25(\mathrm{OH}) \mathrm{D}$ with frailty \\
\hline Sergi et al., 2015 [17] & Italy & Cross-sectional & $n=1567$ & $\begin{array}{c}n=491 \text { pre-frail } \\
(1 \text { positive criterion) } \\
\text { (75.18 } \pm 6.86) \\
\text { male/female: } 166 / 325 \\
n=209 \text { pre-frail } \\
(2 \text { positive criteria) } \\
\text { (77.77 } \pm 7.48) \\
\text { male/female: } 47 / 162\end{array}$ & $\begin{array}{c}n=867 \text { non-frail } \\
\quad(71.68 \pm 5.55) \\
\text { male/female: } 404 / 463\end{array}$ & $\begin{array}{l}\text { Fried's phenotype } \\
\text { (modified) }\end{array}$ & $\begin{array}{l}25(\mathrm{OH}) \mathrm{D} \\
\text { RIA }\end{array}$ & $\begin{array}{l}\downarrow 25(\mathrm{OH}) \mathrm{D} \text { with increasing } \\
\quad \text { pre-frailty severity }\end{array}$ \\
\hline
\end{tabular}


Table 1. Cont.

\begin{tabular}{|c|c|c|c|c|c|c|c|c|}
\hline Study & $\begin{array}{l}\text { First Author } \\
\text { Country }\end{array}$ & Study Design & $\begin{array}{c}\text { Population } \\
\text { (Mean Age } \pm \text { SD) } \\
\text { (years) }\end{array}$ & $\begin{array}{c}\text { Case Population } \\
\text { (Mean Age } \pm \text { SD) (years) }\end{array}$ & $\begin{array}{c}\text { Control Population } \\
\text { (Mean Age } \pm \text { SD) } \\
\text { (years) }\end{array}$ & $\begin{array}{l}\text { Frailty } \\
\text { Criteria }\end{array}$ & $\begin{array}{l}\text { Outcome and Assay } \\
\text { Method }\end{array}$ & Results \\
\hline Shardell et al., 2009 [40] & USA & Cross-sectional & $n=1005$ & $\begin{array}{c}\text { Male: } \\
n=39 \text { frail } \\
(80.8 \pm 7.8) \\
n=151 \text { pre-frail } \\
(76.2 \pm 7.1) \\
\text { Female: } \\
n=64 \text { frail } \\
(82.3 \pm 7.4) \\
n=237 \text { pre-frail } \\
(76.1 \pm 7.5)\end{array}$ & $\begin{array}{c}\text { Male: } \\
n=242 \text { non-frail } \\
(71.7 \pm 5.5) \\
\text { Female: } \\
n=243 \text { non-frail } \\
(72.9 \pm 6.1)\end{array}$ & Fried's phenotype & $\begin{array}{l}25(\mathrm{OH}) \mathrm{D} \\
\text { RIA }\end{array}$ & $\begin{array}{l}\text { Strong associations of } \downarrow \\
\text { 25(OH)D with frailty in men } \\
\text { and weak association } \\
\text { in women }\end{array}$ \\
\hline Shardell et al., 2012 [41] & USA & Cross-sectional & $n=1005$ & $\begin{array}{c}n=100 \text { frail } \\
n=354 \text { pre-frail }\end{array}$ & $n=471$ non-frail & Fried's phenotype & $\begin{array}{l}25(\mathrm{OH}) \mathrm{D} \\
\text { RIA }\end{array}$ & $\begin{array}{l}\text { Participants with } \geq 20 \mathrm{ng} / \mathrm{mL} \text { of } \\
25(\mathrm{OH}) \mathrm{D} \text { had lower prevalence } \\
\text { of frailty }\end{array}$ \\
\hline & & Cross-sectional & & $n=453$ frail & $n=2363$ non-frail & & & $\downarrow 25(\mathrm{OH}) \mathrm{D}$ with frailty \\
\hline Smit et al., 2012 [18] & USA & $\begin{array}{l}\text { Longitudinal } \\
\text { (12-year } \\
\text { follow-up) }\end{array}$ & $n=4731$ & $\begin{array}{c}(73.6 \pm 0.6) \text { male/female: } 140 / 313 \\
n=1915 \text { pre-frail } \\
(72.0 \pm 0.4) \text { male/female: } 705 / 1210\end{array}$ & $\begin{array}{l}(69.4 \pm 0.3) \\
\text { male/female: } \\
\text { 1099/1264 }\end{array}$ & $\begin{array}{l}\text { Fried's phenotype } \\
\text { (modified) }\end{array}$ & $\begin{array}{l}25(\mathrm{OH}) \mathrm{D} \\
\text { RIA }\end{array}$ & $\begin{array}{l}\uparrow \text { Risk of death with frailty and } \\
\text { low serum } 25(\mathrm{OH}) \mathrm{D}\end{array}$ \\
\hline Sousa-Santos et al., 2018 [42] & Portugal & Cross-sectional & $\begin{array}{c}n=1447 \\
\text { (mean 74, range } \\
65-100) \\
\text { male/female: } \\
610 / 873 \\
\end{array}$ & $\begin{array}{c}n=310 \text { frail } \\
n=785 \text { pre-frail }\end{array}$ & $n=352$ non-frail & Fried's phenotype & $\begin{array}{c}25(\mathrm{OH}) \mathrm{D} \\
\text { Electro-chemiluminescence } \\
\text { immunoassay }\end{array}$ & $\begin{array}{l}\downarrow 25(\mathrm{OH}) \mathrm{D} \text { with pre-frailty } \\
\text { and frailty }\end{array}$ \\
\hline Tajar et al., 2013 [43] & UK & Cross-sectional & $n=1504$ male & $\begin{array}{c}n=76 \text { frail } \\
(72.9 \pm 4.7) \\
n=552 \text { pre-frail } \\
(70.9 \pm 5.6)\end{array}$ & $\begin{array}{c}n=876 \text { non-frail } \\
(68.4 \pm 5.5)\end{array}$ & $\begin{array}{l}\text { Fried's phenotype } \\
\text { (modified) } \\
\text { FI (43 items) }\end{array}$ & $\begin{array}{l}25(\mathrm{OH}) \mathrm{D} \\
\text { RIA }\end{array}$ & $\begin{array}{l}\text { Comparable results using } \\
\text { Fried's phenotype and FI. } \\
\downarrow 25(\mathrm{OH}) \mathrm{D} \text { associated } \\
\text { with being pre-frail and frail }\end{array}$ \\
\hline Vogt et al., 2015 [44] & Germany & $\begin{array}{l}\text { Longitudinal } \\
\text { (2.9-year } \\
\text { follow-up) }\end{array}$ & $n=727$ & $\begin{array}{c}n=27 \text { frail } \\
\text { (n.p.) } \\
n=252 \text { pre-frail } \\
\text { (n.p.) } \\
\end{array}$ & $\begin{array}{c}n=448 \text { non-frail } \\
\text { (n.p.) }\end{array}$ & $\begin{array}{l}\text { Fried's phenotype } \\
\text { (modified) }\end{array}$ & $\begin{array}{c}25(\mathrm{OH}) \mathrm{D} \\
\text { Chemiluminescence } \\
\text { immunoassay }\end{array}$ & $\begin{array}{l}\downarrow 25(\mathrm{OH}) \mathrm{D} \text { with incident } \\
\text { pre-frailty and combined } \\
\text { pre-frailty and frailty }\end{array}$ \\
\hline Wang et al., 2014 [45] & China & Cross-sectional & $n=516$ male & $\begin{array}{c}n=174 \text { frail } \\
(81.9 \pm 4.4) \\
n=182 \text { pre-frail } \\
(74.6 \pm 5.2)\end{array}$ & $\begin{array}{c}n=160 \text { non-frail } \\
\quad(72.7 \pm 4.1)\end{array}$ & Fried's phenotype & $\begin{array}{l}25(\mathrm{OH}) \mathrm{D} \\
\text { RIA }\end{array}$ & $\begin{array}{l}\downarrow 25(\mathrm{OH}) \mathrm{D} \text { levels across } \\
\text { frailty categories }\end{array}$ \\
\hline Wilhelm-Leen et al., 2010 [46] & USA & Cross-sectional & $\begin{array}{c}n=5048 \\
\text { male/female: } \\
2469 / 2579\end{array}$ & n.p. & n.p. & $\begin{array}{l}\text { Fried's phenotype } \\
\text { (modified) }\end{array}$ & $\begin{array}{l}25(\mathrm{OH}) \mathrm{D} \\
\text { RIA }\end{array}$ & $\downarrow 25(\mathrm{OH}) \mathrm{D}$ with frailty \\
\hline \multirow[b]{2}{*}{ Wong et al., 2013 [47] } & \multirow[b]{2}{*}{ Australia } & Cross-sectional & $\begin{array}{c}n=4203 \text { male } \\
\quad(70-88)\end{array}$ & n.p. & n.p. & \multirow[b]{2}{*}{ FRAIL scale } & \multirow{2}{*}{$\begin{array}{l}25(\mathrm{OH}) \mathrm{D} \\
\text { Chemiluminescence } \\
\text { immunoassay }\end{array}$} & $\downarrow 25(\mathrm{OH}) \mathrm{D}$ with frailty \\
\hline & & $\begin{array}{l}\text { Longitudinal } \\
\text { (9.2-year } \\
\text { follow-up) }\end{array}$ & $n=1625$ male & & $\begin{array}{l}\mathrm{n}=1625 \text { non-frail } \\
(\text { FRAIL scale }=0 \text { ) }\end{array}$ & & & $\downarrow 25(\mathrm{OH}) \mathrm{D}$ with incident frailty \\
\hline
\end{tabular}

$\uparrow$ : indicates significant increase (although indicated otherwise); $\downarrow$ : indicates significant decrease (although indicated otherwise); 1,25D: 1,25-dihydroxyvitamin D; 25(OH)D: 25-hydroxyvitamin

D; IQR: interquartile range; CI: confidence interval; ECLIA: enhanced chemiluminescence immunoassay; ELISA: enzyme-linked immunosorbent assay; FI: frailty index; FRAIL: Fatigue,

Resistance, Ambulation, Illnesses, \& Loss of Weight; GC/MS: Gas chromatography/mass spectrometry; IQR: interquartile range; LC-MS/MS: Liquid chromatography/tandem mass

spectroscopy; n.p.: not provided; RIA: radioimmunoassay. 
Table 2. Summary of meta-analyses comparing frailty groups with assessment of publication bias and sensitivity analysis. Statistically significant $p$-Values are indicated in bold.

\begin{tabular}{|c|c|c|c|c|c|c|c|c|c|c|c|}
\hline \multirow[t]{2}{*}{ Comparison } & \multirow{2}{*}{$\begin{array}{l}\text { No. of } \\
\text { Studies }\end{array}$} & \multicolumn{2}{|c|}{ Heterogeneity } & \multicolumn{3}{|c|}{ Meta-Analysis } & \multicolumn{2}{|c|}{$\begin{array}{c}\text { Publication Bias } \\
\text { (Egger's Test) }\end{array}$} & \multicolumn{3}{|c|}{ Trim-and-Fill } \\
\hline & & $\mathrm{I}^{2}$ & $p$-Value & SMD & $95 \% \mathrm{CI}$ & $p$-Value & $\mathbf{Z}$ & $p$-Value & SMD & $95 \% \mathrm{CI}$ & $p$-Value \\
\hline \multicolumn{12}{|l|}{ All studies } \\
\hline Frailty vs. non-frailty & 12 & 99.76 & $<0.0001$ & -1.31 & $-2.47,-0.15$ & 0.0271 & -0.4838 & 0.6285 & & & \\
\hline Pre-frailty vs. non-frailty & 12 & 99.83 & $<0.0001$ & -0.79 & $-1.58,-0.003$ & 0.0491 & -0.3639 & 0.7159 & & & \\
\hline Frailty vs. pre-frailty & 9 & 99.61 & $<0.0001$ & -0.82 & $-1.77,0.13$ & 0.09 & 0.4327 & 0.6652 & & & \\
\hline \multicolumn{12}{|c|}{ Sensitivity analysis without Smit et al. (2012) [18] } \\
\hline Frailty vs. non-frailty & 11 & 96.05 & $<0.0001$ & -0.57 & $-0.87,-0.28$ & 0.0002 & -1.3846 & 0.1662 & & & \\
\hline Pre-frailty vs. non-frailty & 11 & 88.35 & $<0.0001$ & -0.27 & $-0.38,-0.17$ & $<0.0001$ & -2.5312 & 0.0114 & -0.21 & $-0.31,-0.12$ & $<0.0001$ \\
\hline Frailty vs. pre-frailty & 8 & 95.55 & $<0.0001$ & -0.46 & $-0.78,-0.14$ & 0.0048 & -0.4693 & 0.6388 & & & \\
\hline
\end{tabular}

CI: confidence interval; SMD: standardized mean difference. 


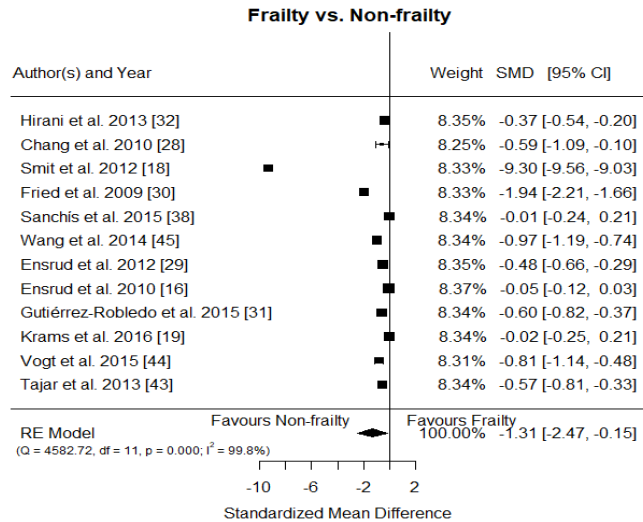

(a)

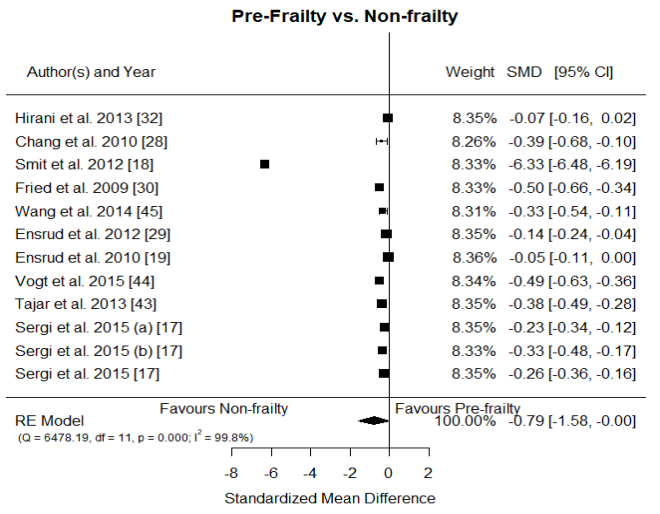

(c)

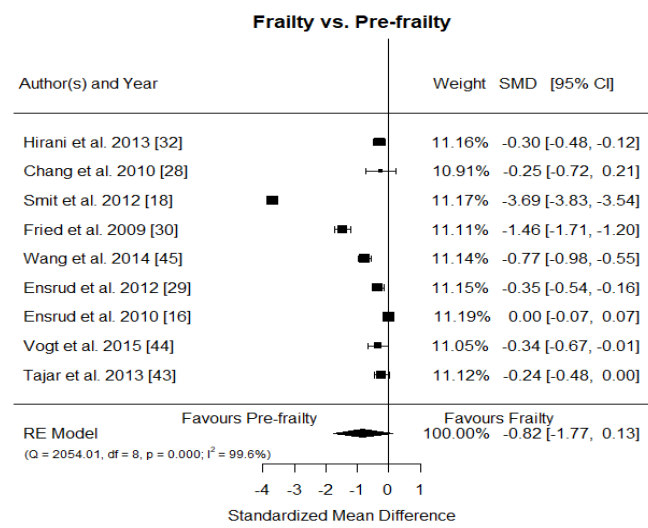

(e)

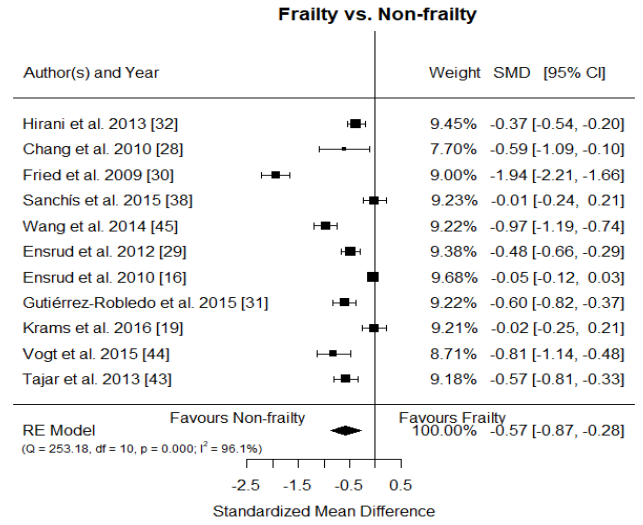

(b)

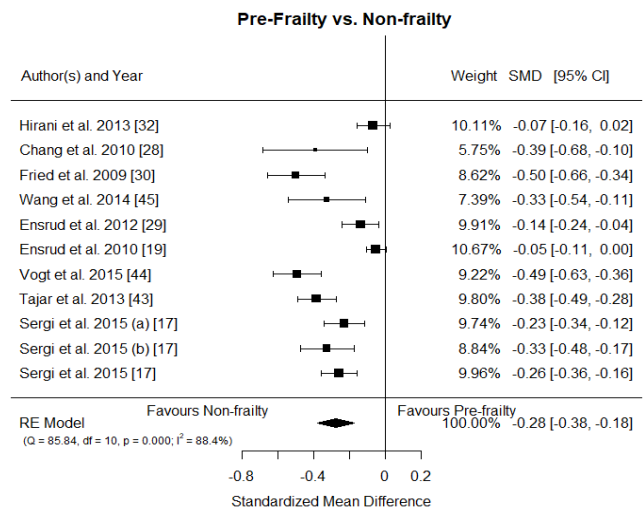

(d)

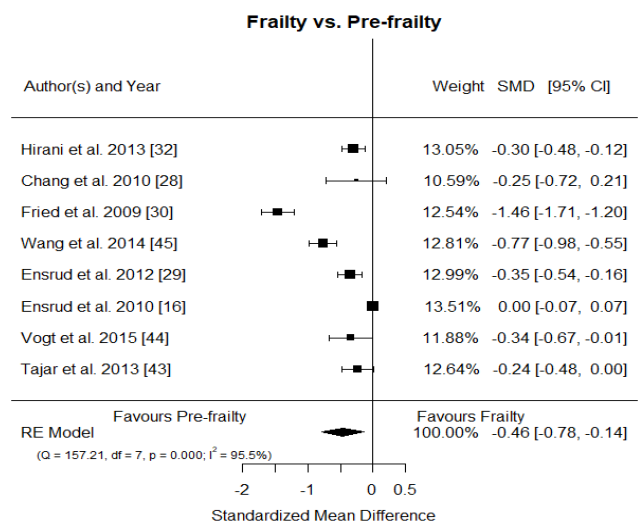

(f)

Figure 2. Forest plots for frailty vs. non-frailty comparison $(\mathbf{a}, \mathbf{b})$, pre-frailty vs. non-frailty comparison $(\mathbf{c}, \mathbf{d})$, and frailty vs. pre-frailty comparison (e,f); forest plots including all studies (a,c,e) and sensitivity analyses excluding Smit et al. [18] (b,d,f) are depicted. In order to respect original data provided by Sergi et al. [17], three populations from this study which differ in the number of positive criteria to classify pre-frail subjects were included in the meta-analyses comparing pre-frailty vs. non-frailty: pre-frail subjects with only one positive frailty criterion were embodied in "Sergi et al. 2015 (a) [17] "; pre-frail subjects with two positive criteria were embodied in "Sergi et al. 2015 (b) [17]"; and all pre-frail subjects (with one and two positive criteria) were embodied in "Sergi et al. 2015 [17]". RE: random-effects. 


\subsection{Meta-Analysis Pre-Frailty Group vs. Non-Frailty Group}

The ten studies included in this meta-analysis evaluated the association between levels of $25(\mathrm{OH}) \mathrm{D}$ and pre-frailty (Figure 2c). In agreement with the original data reported by Sergi et al. [17], all three criteria used to classify subjects as pre-frail, i.e., one, two, or one and two positive Fried's frailty criteria, were considered in the meta-analysis. Heterogeneity was considerably reduced, but still significant $\left(\mathrm{I}^{2}=88.4 \%, p<0.0001\right)$ even after the extreme results by Smit et al. [18] were removed from the meta-analysis (Figure 2d). Also, in this case, a significantly lower level of 25(OH)D was observed in the group of pre-frail subjects when compared to non-frail subjects. The Egger's regression test was not significant, indicating the absence of publication bias.

\subsection{Meta-Analysis Frailty Group vs. Pre-Frailty Group}

The meta-analysis comparing different degrees of frailty included nine studies (Figure 2e). Statistically significant heterogeneity among the studies was observed. Lower concentrations of $25(\mathrm{OH}) \mathrm{D}$ were observed in the frailty group, but this reduction reached statistical significance only when the study by Smit et al. [18] was removed from the comparison (Figure 2f). Egger's test was not significant, suggesting no publication bias.

Meta-regression analyses did not show the presence of any significant effect modification or confounding in any of the comparisons conducted.

\subsection{Sensitivity Analyses}

Considering the high heterogeneity among studies and the fact that the SMDs of the study conducted by Smit et al. [18] were notably discrepant with regard to all other studies included in the three comparisons reported in the forest plots (Figure 2a,c,e), with a much greater reduction of $25(\mathrm{OH}) \mathrm{D}$ in the study groups, we performed sensitivity analyses excluding this particular study in an attempt to reduce heterogeneity and to investigate how much of the overall effect was attributable to it. A summary of the results obtained with and without the study of Smit et al. [18] is reported in Table 2. In general, heterogeneity was reduced after removing this study, although it continued to be significant. Estimates of SMD obtained were lower than in the original analyses, but all of them reached statistical significance, indicating that $25(\mathrm{OH}) \mathrm{D}$ levels decrease while frailty severity increases. The presence of publication bias was only observed in the pre-frailty vs. non-frailty comparison; the estimate of the SMD was slightly changed but still significant after adjusting with the trim-and-fill method. Funnel plots for the three comparisons after excluding the study of Smit et al. [18] are shown in Figure 3. 

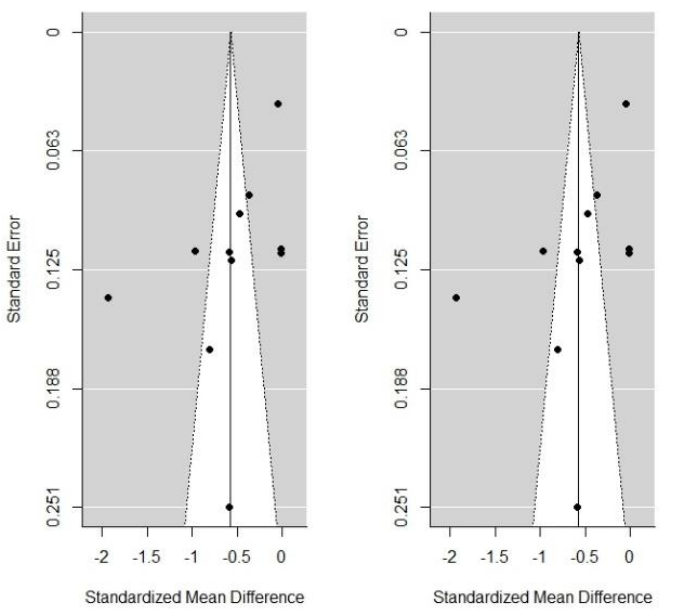

(a)
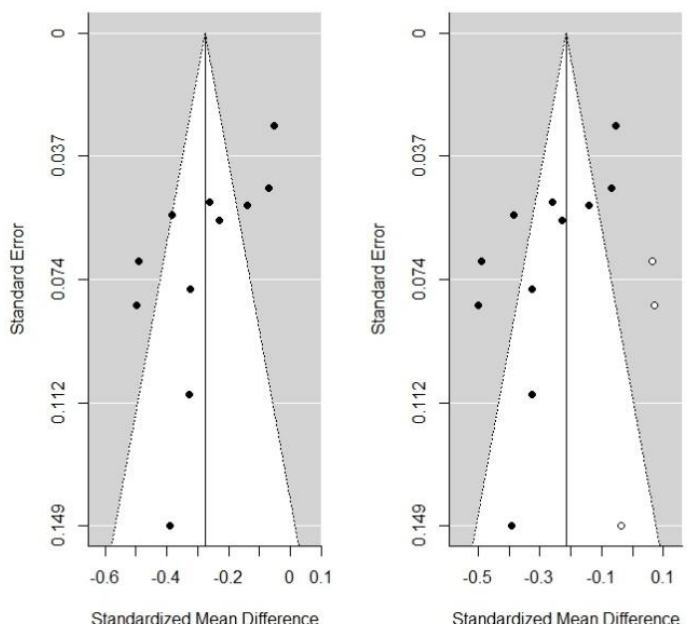

(b)
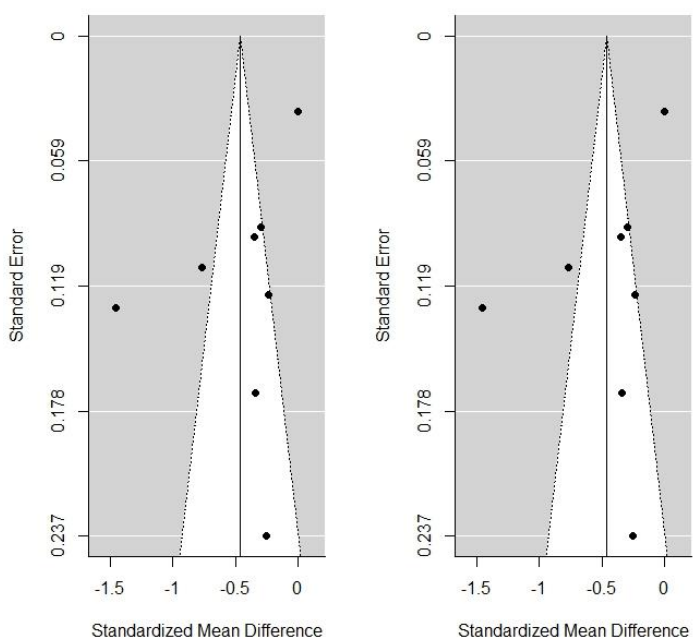

(c)

Figure 3. Funnel plots for 25(OH)D concentrations (sensitivity analyses excluding Smit et al. [18]). (a) frailty vs. non-frailty comparison, (b) pre-frailty vs. non-frailty comparison, and (c) frailty vs. pre-frailty comparison. Original data are shown on the left, and filled-in data in the trim-and-fill method on the right. 


\section{Discussion}

The current and unstoppable ageing of the world's population leads to a constant increase of age-related disabilities, diseases, and frailty. These conditions are attracting the attention of researchers and governments who aim to reduce their effect on public health, mostly through prevention. As previously reported, vitamin D is an age-dependent biomarker associated with type 1 and type 2 diabetes mellitus [48-50], cardiovascular diseases [51,52], various types of cancers [1,53], neurological diseases [4,18], respiratory tract diseases [54], autoimmune diseases [55], and an increased risk of death [56], falls [57], and fractures [8].

Our literature search (26 studies) unequivocally showed an association of low 25(OH)D levels with frailty in almost all studies included in the review. Only one of them [19] failed to find a significant association between $25(\mathrm{OH}) \mathrm{D}$ concentration and frailty. The low proportion of non-frail subjects analyzed $(6.12 \%)$, and the high proportions of both pre-frail (44.21\%) and frail (49.7\%) subjects, notably different to other studies with positive results, may have reduced the possibility of identifying an association between $25(\mathrm{OH}) \mathrm{D}$ and frailty.

Since there are a variety of tools to identify frailty, largely differing in their conceptualization of frailty, we decided to restrict the meta-analyses to those studies employing Fried's criteria in order to increase homogeneity. Our results, which included 13 studies and a total of 20,355 study subjects, showed a clear and inverse relationship between $25(\mathrm{OH}) \mathrm{D}$ levels and frailty severity-as defined by the Fried's phenotype-both in the original analyses and in the sensitivity analyses, which excluded the study by Smit et al. [18]. The exclusion of this study, motivated by results which showed a more pronounced difference between study groups, caused an obvious reduction of heterogeneity among the studies. Besides, the results obtained did not notably modify the original ones, but rather confirmed the association between vitamin D deficiency and frailty; the comparison between frail and pre-frail subjects resulted in a significant difference. The extreme results published by Smit et al. [18] can possibly be explained by the peculiarity of this research, i.e.,: (1) participants were recruited only from institutionalization settings; (2) Fried's frailty criteria were largely modified (i.e., low body mass index instead of unintentional weight loss, muscular weakness determined by a question instead of grip strength measurement, and different questions to identify exhaustion and low physical activity); (3) a large proportion of participants were affected by chronic diseases: $66 \%$ of non-frail, $80 \%$ of pre-frail, and $94 \%$ of frail subjects, and the distribution of the presence of these chronic diseases was significantly different ( $p<0.00001)$ among the three groups of individuals; and (4) the size of the frail group was not balanced with regard the other two groups $(n=453$ frail individuals, $n=1915$ pre-frail, $n=2363$ non-frail individuals). All these conditions contribute to explain the stronger association found in this study and also justify the choice of removing the study in the sensitivity analyses.

Two previous systematic reviews and meta-analyses [21,22] were published on the relationship between vitamin D deficiency and frailty, with results consistent with the current ones, although these studies differ notably from the present one in several aspects of the design and analysis. The study from Zhou and colleagues [22] included only prospective cohort studies $(n=7$, cross-sectional studies were excluded), and compared groups with the most extreme levels of $25(\mathrm{OH}) \mathrm{D}$, calculating the pooled odds ratio of frailty in the lowest versus the highest level of $25(\mathrm{OH}) \mathrm{D}$. Furthermore, in that meta-analysis, cut-off values for defining low and high level categories were different among studies, the number of categories were different (three or four depending on the study), and in some cases, the units used to measure 25(OH)D concentrations were also different. All these discrepancies may have led to a biased interpretation of the results, including an overestimation or an underestimation of the effect. Ju and colleagues [21] conducted a dose response meta-analysis, calculating the pooled risk estimate of frailty for a $25 \mathrm{nmol} / \mathrm{L}$ increment in serum $25(\mathrm{OH}) \mathrm{D}$ concentration in four cross-sectional and six longitudinal cohort studies. In addition, both meta-analyses were affected by remarkable heterogeneity due to the inclusion of studies with non-geriatric subjects (up to 50 years old), and especially by the use of different frailty identification tools. 
The meta-analyses performed in the present study have a number of strengths, including the use of cross-sectional studies only ( $n=13$; for the longitudinal studies, only baseline values were included), the selection of more homogeneous studies by age ( $\geq 60$ years old), and especially the inclusion of studies which identify frailty using only Fried's criteria. The use of absolute values of $25(\mathrm{OH}) \mathrm{D}$ concentration, instead of classes, added specificity to our results. Finally, the two-by-two comparison of non-frail, pre-frail and frail subjects by level of $25(\mathrm{OH}) \mathrm{D}$ provided quantitative estimates of effect which supported the hypothesis of a decrease of $25(\mathrm{OH}) \mathrm{D}$ being associated with the severity of frailty.

In spite of the limitations discussed above, the results of the two previous systematic reviews and meta-analyses point in the same direction of the current study, indicating the presence of a significant association between lower concentrations of $25(\mathrm{OH}) \mathrm{D}$ and frailty. Nevertheless, it is still unclear whether vitamin D deficiency is involved in frailty development or is a consequence of it: frail people present with low muscle strength and have reduced physical activity; hence, they spend less time outdoors, limiting their exposure to sunlight, and their diet is frequently low-quality and unbalanced.

The vitamin D receptor is expressed in the nucleus of muscle cells [58], and vitamin D has been shown to affect muscle cell contractility [59] and increase the de novo synthesis of protein, regulating muscle strength [60]. The number of vitamin D receptors in several organs, including muscle tissues, decreases with age, contributing to reduced muscle strength in later life. Current and previous findings are in accordance with several studies that reported an association between low serum $25(\mathrm{OH}) \mathrm{D}$ levels, poor physical performance and low muscle strength [61,62], and loss of muscle mass and sarcopenia [63], which are individual components of the frailty phenotype. In fact, evidence from randomized clinical trials seems to support a beneficial effect of vitamin D supplements in older adults on muscle strength and function (reviewed in [64]) and on physical performance as assessed by timed up and go test [65]. For this purpose, a daily dose of $1000 \mathrm{IU}$ ( $25 \mu \mathrm{g} / \mathrm{day} ; 1 \mu \mathrm{g}=40 \mathrm{IU})$ appears to be sufficient to obtain significant improvements (enhanced skeletal muscle strength, improvement in physical performance tests, increase in muscle fibers). In contrast, large intermittent doses of vitamin D do not appear to be efficient at improving muscle strength (reviewed in [66]). Falls and fractures are among the main frailty-related health outcomes. In this regard, several meta-analyses $[67,68]$ provided evidence that a supplement of vitamin D combined with calcium (which has a critical structural role, comprising a substantial proportion of the skeleton) results in a $15-20 \%$ reduction of hip or non-vertebral fractures in older participants with vitamin D deficiency, meanwhile supplementation with vitamin $\mathrm{D}$ alone does not seem to prevent fractures or falls, or have clinically meaningful effects on bone mineral density [69].

As the vitamin D receptor is expressed on immune cells (B cells, $\mathrm{T}$ cells, and antigen presenting cells, such as macrophages and dendritic cells), vitamin D plays an important role in the modulation of the innate and adaptive immune response [70,71]. It regulates the production of inflammatory cytokines and immune cells, which are crucial for the pathogenesis of many immune-related diseases [72]. It has been demonstrated that increased concentrations of inflammatory cytokines are associated with frailty status in older adults $[73,74]$, indicating that frail subjects present an additional degree of chronic inflammation with respect to the normal ageing process. Therefore, vitamin D deficiency could act as an intermediate in the relationship between frailty development and an exacerbated inflammatory response. However, evidence from the studies included in a systematic review by Agbalalah et al. [75] did not demonstrate that vitamin D supplementation in adults results in an improvement in circulating inflammatory function biomarkers. Still, this review was not focused on older adults, but mean age of participants in the studies analyzed ranged between 24.8 and 78.8 years. This is an important factor to consider, since only older adults are expected to present a state of low-grade chronic inflammation (the so called "inflammaging"), and hence it is likely that only this population subgroup experiences benefits from vitamin D supplementation.

Vitamin D receptors are also located in the human cortex and hippocampus [76], which are key areas for cognitive function, and their absence has been associated with neurodegenerative dementia such as Alzheimer's disease [77]. A systematic review by Annweiler et al. [78] concluded that the 
association between serum 25(OH)D concentrations and cognitive performance is not yet clearly established, and intervention studies investigating the effect of vitamin D supplementation on cognitive functions have obtained inconsistent results $[79,80]$. The authors suggested that vitamin D insufficiency may negatively affect specific cognitive functions, such as explicit episodic memory, although this conclusion requires further investigations. Physical frailty is often associated with cognitive dysfunction in older adults [81], possibly because of a common underlying pathophysiological and phenotypical basis. The concept of "cognitive frailty" was proposed to emphasize the important role of brain ageing [82]. Cognitive frailty was defined as the simultaneous presence of both physical frailty, operationalized with the Fried's phenotypical model, and cognitive impairment, diagnosed with a Clinical Dementia Rating scale of 0.5 without a concurrent diagnosis of overt dementia or underlying neurological conditions (see Morley [83] for a review). The possibility that vitamin D deficiency is related to cognitive frailty as well as it is to physical frailty should be investigated in future studies.

There are some limitations in our study. Firstly, seven of the studies collected in the systematic review could not be included in the meta-analyses due to the lack of the necessary comparable data $(25(\mathrm{OH}) \mathrm{D}$ mean and SD). Nevertheless, since all these studies reported associations between low concentrations of 25(OH)D and frailty status [27,30,34,39-41,46], it is likely that present data are not affected by a selection bias. Secondly, sample size was not balanced among the different frailty groups, being frail subjects underrepresented (14\%) with regard to pre-frail (42\%) and non-frail participants $(44 \%)$. Thirdly, the number of longitudinal cohort studies was considered too low to perform a meta-analysis including only prospective data to study the relationship between $25(\mathrm{OH}) \mathrm{D}$ levels and frailty at follow up, and these studies employed different frailty identification tools. These latter issues, however, may have introduced a non-directional loss of precision and quality rather that generating biased results. Fourthly, heterogeneity among studies was quite high, even in the sensitivity analyses, although it was only a quantitative heterogeneity since almost all studies showed lower level of $25(\mathrm{OH}) \mathrm{D}$ in frail and pre-frail subjects. Finally, another limitation may be the language restriction of the literature search; we reviewed only studies written in English or Spanish and may have missed informative studies in other languages. Nevertheless, this issue does not seem to affect met-estimates, given the lack of publication bias demonstrated by Egger's regression test results.

\section{Conclusions}

In conclusion, the results of the present study indicate the presence of an inverse association between serum $25(\mathrm{OH}) \mathrm{D}$ concentration and frailty severity, as defined by the Fried's phenotype. In view of the current and previous results, considering that vitamin D supplementation is safe and inexpensive, and beneficial effects on the muscular performance have been demonstrated, interventional studies testing the possible benefits of vitamin D supplementation in older adults to prevent/palliate frailty should be considered. A further challenging question is whether vitamin D supplementation in frail subjects may also reduce the negative health outcomes associated with frailty, including dependency and mortality, and whether it may have some effect on cognitive frailty.

Supplementary Materials: The following are available online at http://www.mdpi.com/2072-6643/12/8/2286/s1, Table S1. Quality score criteria of the studies included in the meta-analyses.

Author Contributions: E.P., B.L., J.P.T. and J.F.-T. conceptualized and designed the study. D.M.-P., V.V., and M.S.-F. conducted the literature search and screening, data collection and contacts with authors. S.P., S.C., and S.B. carried out the statistical analyses. D.M.-P., B.L., and V.V. wrote the manuscript draft. All authors have read and agreed to the published version of the manuscript.

Funding: This research was funded by Xunta de Galicia [ED431B 2019/02]; Ministerio de Educación, Cultura y Deporte [BEAGAL18/00142 to V.V, PRX19/00353 to B.L.]; and Deputación Provincial de A Coruña [to D.M.-P. and M.S.-F.]

Acknowledgments: We want to express our gratitude to K. Ensrud (Center for Chronic Disease Outcomes Research, VA Medical Center); J. Sanchís (Department of Cardiology, Hospital Clínico Universitario, Valencia, School of Medicine, University of Valencia); and S. Vogt (Institute of Epidemiology II, Helmholtz Zentrum München, German Research Center for Environmental Health) for sending us their data. 
Conflicts of Interest: The authors declare no conflict of interest.

\section{References}

1. Hossein-nezhad, A.; Holick, M.F. Vitamin D for Health: A Global Perspective. Mayo Clin. Proc. 2013, 88, 720-755. [CrossRef] [PubMed]

2. Cardus, A.; Panizo, S.; Encinas, M.; Dolcet, X.; Gallego, C.; Aldea, M.; Fernandez, E.; Valdivielso, J.M. 1,25-Dihydroxyvitamin D3 regulates VEGF production through a vitamin D response element in the VEGF promoter. Atherosclerosis 2009, 204, 85-89. [CrossRef] [PubMed]

3. Richart, T.; Li, Y.; Staessen, J.A. Renal Versus Extrarenal Activation of Vitamin D in Relation to Atherosclerosis, Arterial Stiffening, and Hypertension. Am. J. Hypertens. 2007, 20, 1007-1015. [CrossRef] [PubMed]

4. Holick, M.F. Vitamin D: Extraskeletal Health. Rheum. Dis. Clin. N. Am. 2012, 38, 141-160. [CrossRef]

5. Ross, A.C.; Manson, J.E.; Abrams, S.A.; Aloia, J.F.; Brannon, P.M.; Clinton, S.K.; Durazo-Arvizu, R.A.; Gallagher, J.C.; Gallo, R.L.; Jones, G.; et al. The 2011 Dietary Reference Intakes for Calcium and Vitamin D: What Dietetics Practitioners Need to Know. J. Am. Diet. Assoc. 2011, 111, 524-527. [CrossRef]

6. Ganji, V.; Zhang, X.; Tangpricha, V. Serum 25-Hydroxyvitamin D Concentrations and Prevalence Estimates of Hypovitaminosis D in the U.S. Population Based on Assay-Adjusted Data. J. Nutr. 2012, 142, 498-507. [CrossRef]

7. Greene-Finestone, L.; Berger, C.; De Groh, M.; Hanley, D.; Hidiroglou, N.; Sarafin, K.; Poliquin, S.; Krieger, J.; Richards, J.; Goltzman, D.; et al. 25-Hydroxyvitamin D in Canadian adults: Biological, environmental, and behavioral correlates. Osteoporos. Int. 2011, 22, 1389-1399. [CrossRef]

8. Lips, P. Vitamin, D. Deficiency and Secondary Hyperparathyroidism in the Elderly: Consequences for Bone Loss and Fractures and Therapeutic Implications. Endocr. Rev. 2001, 22, 477-501. [CrossRef]

9. Souberbielle, J.C.; Cormier, C.; Kindermans, C.; Gao, P.; Cantor, T.; Forette, F.; Baulieu, E.E. Vitamin D status and redefining serum parathyroid hormone reference range in the elderly. J. Clin. Endocrinol. Metab. 2001, 86, 3086-3090. [CrossRef]

10. De Jongh, R.T.; Van Schoor, N.M.; Lips, P. Changes in vitamin D endocrinology during aging in adults. Mol. Cell. Endocrinol. 2017, 453, 144-150. [CrossRef]

11. Morley, J.E.; Malmstrom, T.K. Frailty, Sarcopenia, and Hormones. Endocrinol. Metab. Clin. N. Am. 2013, 42, 391-405. [CrossRef] [PubMed]

12. Fried, L.; Tangen, C.; Walston, J.; Newman, A.; Hirsch, C.; Gottdiener, J.; Seeman, T.; Tracy, R.; Kop, W.J.; Burke, G.; et al. Frailty in older adults: Evidence for a phenotype. J. Gerontol. A Biol. Med. Sci. 2001, 56, M146-M156. [CrossRef] [PubMed]

13. Mitnitski, A.; Mogilner, A.; Rockwood, K. Accumulation of Deficits as a Proxy Measure of Aging. Sci. World 2001, 1, 323-336. [CrossRef] [PubMed]

14. Collard, R.; Boter, H.; Schoevers, R.; Voshaar, R. Prevalence of frailty in community-dwelling older persons: A systematic review. J. Am. Geriatr. Soc. 2012, 60, 1487-1492. [CrossRef]

15. Schöttker, B.; Rothenbacher, D.; Perna, L.; Müller, H.; Brenner, H. Serum 25-hydroxyvitamin D levels and incident diabetes mellitus type 2: A competing risk analysis in a large population-based cohort of older adults. Eur. J. Epidemiol. 2013, 28, 267-275. [CrossRef]

16. Ensrud, K.E.; Ewing, S.K.; Fredman, L.; Hochberg, M.C.; Cauley, J.A.; Hillier, T.A.; Cummings, S.R.; Yaffe, K.; Cawthon, P.M. Circulating 25-Hydroxyvitamin D Levels and Frailty Status in Older Women. J. Clin. Endocrinol. Metab. 2010, 95, 5266-5273. [CrossRef]

17. Sergi, G.; Veronese, N.; Fontana, L.; De Rui, M.; Bolzetta, F.; Zambon, S.; Corti, M.C.; Baggio, G.; Toffanello, E.D.; Crepaldi, G.; et al. Pre-frailty and risk of cardiovascular disease in elderly men and women: The Pro.V.A. Study. J. Am. Coll. Cardiol. 2015, 65, 976-983. [CrossRef]

18. Smit, E.; Crespo, C.J.; Michael, Y.; Ramirez-Marrero, F.A.; Brodowicz, G.R.; Bartlett, S.; Andersen, R.E. The effect of vitamin D and frailty on mortality among non-institutionalized US older adults. Eur. J. Clin. Nutr. 2012, 66, 1024-1028. [CrossRef]

19. Krams, T.; Cesari, M.; Guyonnet, S.; Abellan van Kan, G.; Cantet, C.; Vellas, B.; Rolland, Y. Is the 25-hydroxy-vitamin D serum concentration a good marker of frailty? J. Nutr. Health Aging 2016, 20, 1034-1039. [CrossRef] 
20. Schoufour, J.D.; Echteld, M.A.; Boonstra, A.; Groothuismink, Z.M.A.A.; Evenhuis, H.M. Biochemical measures and frailty in people with intellectual disabilities. Age Ageing 2016, 45, 142-148. [CrossRef]

21. Ju, S.Y.; Lee, J.Y.; Kim, D.H. Low 25-hydroxyvitamin D levels and the risk of frailty syndrome: A systematic review and dose-response meta-analysis. BMC Geriatr. 2018, 18, 206. [CrossRef] [PubMed]

22. Zhou, J.; Huang, P.; Liu, P.; Hao, Q.; Chen, S.; Dong, B.; Wang, J. Association of vitamin D deficiency and frailty: A systematic review and meta-analysis. Maturitas 2016, 94, 70-76. [CrossRef] [PubMed]

23. Moher, D.; Liberati, A.; Tetzlaff, J.; Altman, D.G.; Altman, D.; Antes, G.; Atkins, D.; Barbour, V.; Barrowman, N.; Berlin, J.A.; et al. Preferred reporting items for systematic reviews and meta-analyses: The PRISMA statement. J. Clin. Epidemiol. 2009, 62, 1006-1012. [CrossRef] [PubMed]

24. Dersimonian, R.; Laird, N. Meta-Analysis in Clinical Trials *. Control. Clin. Trials 1986, 7, 177-188. [CrossRef]

25. Viechtbauer, W. Conducting Meta-Analyses in R with the metafor Package. J. Stat. Softw. 2010, 36, 1-48. [CrossRef]

26. Alvarez-Ríos, A.I.; Guerrero, J.M.; García-garcía, F.J.; Rodríguez-mañas, L.; Medrano-campillo, P.; De, M.A.; Lanza, T.; Alvarez-sánchez, N.; Carrillo-vico, A. Associations between frailty and serum N-terminal propeptide of type I procollagen and 25-hydroxyvitamin D in older Spanish women: The Toledo Study for Healthy Aging. EXG 2015, 69, 79-84. [CrossRef]

27. Álvarez-Sánchez, N.; Álvarez-Ríos, A.I.; Guerrero, J.M.; García-García, F.J.; Rodríguez-Mañas, L.; Cruz-Chamorro, I.; Lardone, P.J.; Carrillo-Vico, A. Homocysteine levels are associated with bone resorption in pre-frail and frail Spanish women: The Toledo Study for Healthy Aging. Exp. Gerontol. 2018, 108, 201-208. [CrossRef]

28. Chang, C.-I.; Chan, D.-C.D.; Kuo, K.-N.; Hsiung, C.A.; Chen, C.-Y. Vitamin D insufficiency and frailty syndrome in older adults living in a Northern Taiwan community. Arch. Gerontol. Geriatr. 2010, 50 (Suppl. 1), S17-S21. [CrossRef]

29. Ensrud, K.; Blackwell, T.; Cauley, J.; Cummings, S.; Barrett-Connor, J.; Dam, T.; Hoffman, A.; Shikany, J.; Lane, N.; Stefanick, M.; et al. Circulating 25-hydroxyvitamin D Levels and Frailty Status in Older Men: The Osteoporotic Fractures in Men Study. J. Am. Geriatr. Soc. 2012, 59, 101-106. [CrossRef]

30. Fried, L.; Xue, Q.; Cappola, A.; Ferrucci, L.; Chaves, P.; Varadhan, R.; Guralnik, J.; Leng, S.; Semba, R.; Walston, J.; et al. Nonlinear multisystem physiological dysregulation associated with frailty in older women: Implications for etiology and treatment. J. Gerontol. A Biol. Med. Sci. 2009, 64, 1049-1057. [CrossRef]

31. Gutiérrez-Robledo, L.M.; Ávila-funes, J.A.; Amieva, H.; Meillon, C.; Acosta, J.L.; Navarrete-reyes, A.P.; Muñoz-valle, J.F.; Torres-carrillo, N.M.; Miguel, L.; Ávila-funes, J.A.; et al. Association of low serum 25-hydroxyvitamin D levels with the frailty syndrome in Mexican community-dwelling elderly. Aging Male 2016, 19, 58-63. [CrossRef] [PubMed]

32. Hirani, V.; Naganathan, V.; Cumming, R.G.; Blyth, F.; Le Couteur, D.G.; Handelsman, D.J.; Waite, L.M.; Seibel, M.J. Associations between frailty and serum 25-hydroxyvitamin D and 1,25-dihydroxyvitamin D concentrations in older australian men: The concord health and ageing in men project. J. Gerontol. Ser. A Biol. Sci. Med. Sci. 2013, 68, 1112-1121. [CrossRef] [PubMed]

33. Kojima, G.; Tanabe, M. Frailty is Highly Prevalent and Associated with Vitamin D Deficiency in Male Nursing Home Residents. J. Am. Geriatr. Soc. 2016, 64, e33-e35. [CrossRef] [PubMed]

34. Michelon, E.; Blaum, C.; Semba, R.D.; Xue, Q.-L.; Ricks, M.O.; Fried, L.P. Vitamin and carotenoid status in older women: Associations with the frailty syndrome. J. Gerontol. A Biol. Sci. Med. Sci. 2006, 61, 600-607. [CrossRef] [PubMed]

35. Navarro-Martínez, R.; Fernández-garrido, J.; Buigues, C.; Martinez-martinez, M.; Cantero-díaz, L.; Santamaría-carrillo, Y.; Serra-catalá, N.; Peris, C.; Cauli, O. Serum vitamin D and functional impairment in octogenarian women. Appl. Nurs. Res. 2016, 30, e10-e14. [CrossRef]

36. Pabst, G.; Zimmermann, A.K.; Huth, C.; Koenig, W.; Ludwig, T.; Zierer, A.; Peters, A.; Thorand, B. Association of low 25-hydroxyvitamin D levels with the frailty syndrome in an aged population: Results from the KORA-Age Augsburg study. J. Nutr. Health Aging 2015, 19, 258-264. [CrossRef]

37. Puts, M.T.E.; Visser, M.; Twisk, J.W.R.; Deeg, D.J.H.; Lips, P. Endocrine and inflammatory markers as predictors of frailty. Clin. Endocrinol. 2005, 63, 403-411. [CrossRef]

38. Sanchis, J.; Núñez, E.; Ruiz, V.; Bonanad, C.; Fernández, J.; Cauli, O.; García-blas, S.; Mainar, L.; Valero, E.; Rodríguez-borja, E.; et al. Usefulness of Clinical Data and Biomarkers for the Identi fi cation of Frailty After Acute Coronary Syndromes. Can. J. Cardiol. 2015, 31, 1-7. [CrossRef] 
39. Semba, R.D.; Bartali, B.; Zhou, J.; Blaum, C.; Ko, C.-W.; Fried, L.P. Low Serum Micronutrient Concentrations Predict Frailty Among Older Women Living in the Community. J. Gerontol. Ser. A Biol. Sci. Med. Sci. 2006, 61, 594-599. [CrossRef]

40. Shardell, M.; Hicks, G.E.; Miller, R.R.; Kritchevsky, S.; Andersen, D.; Bandinelli, S.; Cherubini, A.; Ferrucci, L. Association of low vitamin D levels with the frailty syndrome in men and women. J. Gerontol. A. Biol. Sci. Med. Sci. 2009, 64, 69-75. [CrossRef]

41. Shardell, M.; D’Adamo, C.; Alley, D.E.; Miller, R.R.; Hicks, G.E.; Milaneschi, Y.; Semba, R.D.; Cherubini, A.; Bandinelli, S.; Ferrucci, L. Serum 25-hydroxyvitamin D, transitions between frailty states, and mortality in older adults: The invecchiare in Chianti study. J. Am. Geriatr. Soc. 2012, 60, 256-264. [CrossRef] [PubMed]

42. Sousa-Santos, A.R.; Afonso, C.; Santos, A.; Borges, N.; Moreira, P.; Padrão, P.; Fonseca, I.; Amaral, T.F. The association between $25(\mathrm{OH}) \mathrm{D}$ levels, frailty status and obesity indices in older adults. PLoS ONE 2018, 13, 1-16. [CrossRef] [PubMed]

43. Tajar, A.; Lee, D.M.; Pye, S.R.; O'Connell, M.D.L.; Ravindrarajah, R.; Gielen, E.; Boonen, S.; Vanderschueren, D.; Pendleton, N.; Finn, J.D.; et al. The association of frailty with serum 25-hydroxyvitamin d and parathyroid hormone levels in older european men. Age Ageing 2013, 42, 352-359. [CrossRef] [PubMed]

44. Vogt, S.; Decke, S.; Gala, T.; De Las, H.; Linkohr, B.; Koenig, W.; Ladwig, K.H.; Peters, A.; Thorand, B. Prospective association of vitamin $\mathrm{D}$ with frailty status and all-cause mortality in older adults: Results from the KORA-Age Study. Prev. Med. 2015, 73, 40-46. [CrossRef]

45. Wang, Y.; Wang, Y.; Zhan, J.; Tang, Z.; Huang, W.; Tan, P.; Gao, S.; Ma, C.; Jian, Z.; Liu, Y. Vitamin D Binding Protein Affects the Correlation of $25(\mathrm{OH}) \mathrm{D}$ and Frailty in the Older Men. Int. J. Endocrinol. 2014, 2014, 543783. [CrossRef]

46. Wilhelm-Leen, E.R.; Hall, Y.N.; Deboer, I.H.; Chertow, G.M. Vitamin D deficiency and frailty in older Americans. J. Intern. Med. 2010, 268, 171-180. [CrossRef] [PubMed]

47. Wong, Y.Y.E.; McCaul, K.A.; Yeap, B.B.; Hankey, G.J.; Flicker, L. Low vitamin D status is an independent predictor of increased frailty and all-cause mortality in older men: The health in men study. J. Clin. Endocrinol. Metab. 2013, 98, 3821-3828. [CrossRef]

48. Mitri, J.; Pittas, A.G. Vitamin D and type 2 diabetes: A systematic review. Eur. J. Clin. Nutr. 2011, 65, 1005-1015. [CrossRef]

49. Pittas, A.; Nelson, J.; Hillmann, W.; Garganta, C.; Nathan, D.; Hu, F.; Dawson-Hughes, B.; Diabetes Prevention Program Research Group. Plasma 25-Hydroxyvitamin D and Progression to Diabetes in Patients at Risk for Diabetes. An ancillary analysis in the Diabetes Prevention Program. Diabetes Care 2012, 35, 565-573. [CrossRef]

50. Ozfirat, Z.; Chowdhury, T.A. Vitamin D deficiency and type 2 diabetes. Postgrad. Med. J. 2010, 86, 18-25. [CrossRef]

51. Giovannucci, E.; Liu, Y.; Hollis, B.; Rimm, E. A Prospective Study of 25-Hydroxy-Vitamin D and Risk of Myocardial Infarction in Men. Arch. Intern. Med. 2008, 168, 1174-1180. [CrossRef] [PubMed]

52. Judd, S.E.; Tangpricha, V. Vitamin D Deficiency and Risk for Cardiovascular Disease. Am. J. Med. Sci. 2009, 338, 40-44. [CrossRef] [PubMed]

53. Shui, I.M.; Mucci, L.A.; Kraft, P.; Tamimi, R.M.; Lindstrom, S.; Penney, K.L.; Nimptsch, K.; Hollis, B.W.; Dupre, N.; Platz, E.A.; et al. Vitamin D—Related Genetic Variation, Plasma Vitamin D, and Risk of Lethal Prostate Cancer: A Prospective Nested Case-Control Study. J. Natl. Cancer Inst. 2012, 104, 690-699. [CrossRef] [PubMed]

54. Sabetta, J.R.; Depetrillo, P.; Cipriani, R.J.; Smardin, J.; Burns, L.A.; Marie, L. Serum 25-Hydroxyvitamin D and the Incidence of Acute Viral Respiratory Tract Infections in Healthy Adults. PLoS ONE 2010, 5, e11088. [CrossRef] [PubMed]

55. Agmon-levin, N.; Kivity, S.; Tzioufas, A.G.; López, M.; Rozman, B.; Efes, I.; Shapira, Y.; Shamis, A.; Amital, H.; Youinou, P.; et al. Low levels of vitamin-D are associated with neuropathy and lymphoma among patients with Sjögren's syndrome. J. Autoimmun. 2012, 39, 234-239. [CrossRef] [PubMed]

56. Pilz, S.; Tomaschitz, A.; März, W.; Drechsler, C.; Ritz, E.; Zittermann, A.; Cavalier, E.; Pieber, T.R.; Lappe, J.M.; Grant, W.B.; et al. Vitamin D, cardiovascular disease and mortality. Clin. Endocrinol. 2011, 75, 575-584. [CrossRef] 
57. Snijder, M.B.; Schoor, N.M.; Van Pluijm, S.M.F.; Dam, R.M.; Van Visser, M. Vitamin D Status in Relation to One-Year Risk of Recurrent Falling in Older Men and Women. J. Clin. Endocrinol. Metab. 2006, 91, 2980-2985. [CrossRef]

58. Bischoff-Ferrari, H.A.; Borchers, M.; Gudat, F.; Dürmüller, U.; Stähelin, H.B.; Dick, W. Vitamin D Receptor Expression in Human Muscle Tissue Decreases with Age. J. Bone Miner. Res. 2004, 19, 265-269. [CrossRef]

59. Marcinkowska, E. A run for a membrane vitamin d receptor. NeuroSignals 2001, 10, 341-349. [CrossRef]

60. Pfeifer, M.; Begerow, B.; Minne, H.W. Vitamin D and Muscle Function. Osteoporos. Int. 2002, 13, $187-194$. [CrossRef]

61. Stewart, J.W.; Lee Alekel, D.; Ritland, L.M.; Van Loan, M.; Gertz, E.; Genschel, U. Serum 25-hydroxyvitamin $\mathrm{D}$ is related to indicators of overall physical fitness in healthy postmenopausal women. Menopause 2009, 16, 1093-1101. [CrossRef]

62. Wicherts, I.S.; Van Schoor, N.M.; Boeke, A.J.P.; Visser, M.; Deeg, D.J.H.; Smit, J.; Knol, D.L.; Lips, P. Vitamin D status predicts physical performance and its decline in older persons. J. Clin. Endocrinol. Metab. 2007, 92, 2058-2065. [CrossRef]

63. Visser, M.; Deeg, D.; Lips, P. Low Vitamin D and High Parathyroid Hormone Levels as Determinants of Loss of Muscle Strength and Muscle Mass (Sarcopenia): The Longitudinal Aging Study Amsterdam. J. Clin. Endocrinol. Metab. 2003, 88, 5766-5772. [CrossRef] [PubMed]

64. Rejnmark, L. Effects of vitamin D on muscle function and performance: A review of evidence from randomized controlled trials. Ther. Adv. Chronic Dis. 2011, 2, 25-37. [CrossRef]

65. Dewansingh, P.; Melse-Boonstra, A.; Krijnen, W.P.; Van der Schans, C.P.; Jager-Wittenaar, H.; Van den Heuvel, E.G.H.M. Supplemental protein from dairy products increases body weight and vitamin D improves physical performance in older adults: A systematic review and meta-analysis. Nutr. Res. 2018, 49, 1-22. [CrossRef] [PubMed]

66. Halfon, M.; Phan, O.; Teta, D. Vitamin D: A Review on Its Effects on Muscle Strength, the Risk of Fall, and Frailty. Biomed. Res. Int. 2015, 2015, 1-11. [CrossRef] [PubMed]

67. Avenell, A.; Mak, J.C.; O'Connell, D. Vitamin D and vitamin D analogues for preventing fractures in post-menopausal women and older men. Cochrane Database Syst. Rev. 2014, 1465-1858. [CrossRef] [PubMed]

68. Bolland, M.J.; Grey, A.; Gamble, G.D.; Reid, I.R. The effect of vitamin D supplementation on skeletal, vascular, or cancer outcomes: A trial sequential meta-analysis. Lancet Diabetes Endocrinol. 2014, 2, 307-320. [CrossRef]

69. Bolland, M.J.; Grey, A.; Avenell, A. Effects of vitamin D supplementation on musculoskeletal health: A systematic review, meta-analysis, and trial sequential analysis. Lancet Diabetes Endocrinol. 2018, 6, 847-858. [CrossRef]

70. Holick, M.F.; Binkley, N.C.; Bischoff-Ferrari, H.A.; Gordon, C.M.; Hanley, D.A.; Heaney, R.P.; Murad, M.H.; Weaver, C.M. Evaluation, Treatment, and Prevention of Vitamin D Deficiency: An Endocrine Society Clinical Practice Guideline. J. Clin. Endocrinol. Metab. 2011, 96, 1911-1930. [CrossRef]

71. Etten, E.; Van Mathieu, C. Immunoregulation by 1,25-dihydroxyvitamin D3: Basic concepts. J. Steroid Biochem. Mol. Biol. 2005, 97, 93-101. [CrossRef]

72. Liu, W.; Zhang, L.; Xu, H.-J.; Li, Y.; Hu, C.-M.; Yang, J.-Y.; Sun, M.-Y. The Anti-Inflammatory Effects of Vitamin D in Tumorigenesis. Int. J. Mol. Sci. 2018, 19, 2736. [CrossRef]

73. Marcos-Pérez, D.; Sánchez-Flores, M.; Maseda, A.; Lorenzo-López, L.; Millán-Calenti, J.; Gostner, J.; Fuchs, D.; Pásaro, E.; Laffon, B.; Valdiglesias, V. Frailty in Older Adults Is Associated With Plasma Concentrations of Inflammatory Mediators but Not With Lymphocyte Subpopulations. Front. Immunol. 2018, 9, 1-9. [CrossRef]

74. Soysal, P.; Stubbs, B.; Lucato, P.; Luchini, C.; Solmi, M.; Peluso, R.; Sergi, G.; Isik, A.; Manzato, E.; Maggi, S.; et al. Inflammation and frailty in the elderly: A systematic review and meta-analysis. Ageing Res. Rev. 2016, 31,1-8. [CrossRef] [PubMed]

75. Agbalalah, T.; Hughes, S.F.; Freeborn, E.J.; Mushtaq, S. Impact of vitamin D supplementation on endothelial and inflammatory markers in adults: A systematic review. J. Steroid Biochem. Mol. Biol. 2017, 173, 292-300. [CrossRef] [PubMed]

76. Kalueff, A.V.; Tuohimaa, P. Neurosteroid hormone vitamin D and its utility in clinical nutrition. Curr. Opin. Clin. Nutr. Metab. Care 2007, 10, 12-19. [CrossRef] [PubMed]

77. Sutherland, M.K.; Somerville, M.J.; Yoong, L.K.K.; Bergeron, C.; Haussler, M.R.; Crapper McLachlan, D.R. Reduction of vitamin D hormone receptor mRNA levels in Alzheimer as compared to Huntington hippocampus: Correlation with calbindin-28k mRNA levels. Mol. Brain Res. 1992, 13, 239-250. [CrossRef] 
78. Annweiler, C.; Allali, G.; Allain, P.; Bridenbaugh, S.; Schott, A.M.; Kressig, R.W.; Beauchet, O. Vitamin D and cognitive performance in adults: A systematic review. Eur. J. Neurol. 2009, 16, 1083-1089. [CrossRef]

79. Przybelski, R.; Agrawal, S.; Krueger, D.; Engelke, J.A.; Walbrun, F.; Binkley, N. Rapid correction of low vitamin D status in nursing home residents. Osteoporos. Int. 2008, 19, 1621-1628. [CrossRef]

80. Rondanelli, M.; Trotti, R.; Opizzi, A.; Solerte, S.B. Relationship among nutritional status, pro/antioxidant balance and cognitive performance in a group of free-living healthy elderly. Minerva Med. 2007, 98, 639-645.

81. Kelaiditi, E.; Canevelli, M.; Andrieu, S.; Del Campo, N.; Soto, M.E.; Vellas, B.; Cesari, M. Frailty Index and Cognitive Decline in Alzheimer's Disease: Data from the Impact of Cholinergic Treatment USe Study. J. Am. Geriatr. Soc. 2016, 64, 1165-1170. [CrossRef] [PubMed]

82. Kelaiditi, E.; Cesari, M.; Cavanelli, M.; Abellan van Kan, G.; Ousset, P.; Gillette-Guyonnet, S.; Ritz, P.; Duveau, F.; Soto, M.; Provencher, V.; et al. Cognitive frailty: Rational and definition from an (I.A.N.A/I.A.G.G) international consensus group. J. Nutr. Health Aging 2013, 17, 262. [CrossRef] [PubMed]

83. Morley, J.E. Cognitive frailty: A new geriatric syndrome? Eur. Geriatr. Med. 2015, 6, 408-411. [CrossRef]

(C) 2020 by the authors. Licensee MDPI, Basel, Switzerland. This article is an open access article distributed under the terms and conditions of the Creative Commons Attribution (CC BY) license (http://creativecommons.org/licenses/by/4.0/). 